\title{
On the Discrete Spectrum of a Model Operator in Fermionic Fock Space
}

\author{
Zahriddin Muminov, ${ }^{1,2}$ Fudziah Ismail, ${ }^{1,3}$ Zainidin Eshkuvatov, \\ and Jamshid Rasulov ${ }^{4}$ \\ ${ }^{1}$ Department of Mathematics, Faculty of Science, University Putra Malaysia, Malaysia \\ ${ }^{2}$ Samarkand State University, Samarkand, Uzbekistan \\ ${ }^{3}$ Institute For Mathematical Research, UPM, Malaysia \\ ${ }^{4}$ Academic Lyceum Number One under Samarkand Institute of Economics and Service, Samarkand, Uzbekistan
}

Correspondence should be addressed to Zahriddin Muminov; zimuminov@gmail.com

Received 19 January 2013; Revised 15 April 2013; Accepted 21 April 2013

Academic Editor: Pavel Kurasov

Copyright (C) 2013 Zahriddin Muminov et al. This is an open access article distributed under the Creative Commons Attribution License, which permits unrestricted use, distribution, and reproduction in any medium, provided the original work is properly cited.

\begin{abstract}
We consider a model operator $H$ associated with a system describing three particles in interaction, without conservation of the number of particles. The operator $H$ acts in the direct sum of zero-, one-, and two-particle subspaces of the fermionic Fock space $\mathscr{F}_{a}\left(L^{2}\left(\mathbb{T}^{3}\right)\right)$ over $L^{2}\left(\mathbb{T}^{3}\right)$. We admit a general form for the "kinetic" part of the Hamiltonian $H$, which contains a parameter $\gamma$ to distinguish the two identical particles from the third one. (i) We find a critical value $\gamma^{*}$ for the parameter $\gamma$ that allows or forbids the Efimov effect (infinite number of bound states if the associated generalized Friedrichs model has a threshold resonance) and we prove that only for $\gamma<\gamma^{*}$ the Efimov effect is absent, while this effect exists for any $\gamma>\gamma^{*}$. (ii) In the case $\gamma>\gamma^{*}$, we also establish the following asymptotics for the number $N(z)$ of eigenvalues of $H$ below $z<E_{\min }=\inf \sigma_{\text {ess }}(H)$ : $\lim _{z \rightarrow E_{\min }}\left(N(z) /|\log | E_{\min }-z||\right)=\mathscr{U}_{0}(\gamma)\left(\mathcal{U}_{0}(\gamma)>0\right)$, for all $\gamma>\gamma^{*}$.
\end{abstract}

\section{Introduction}

In the spectral theory of the continuous and lattice threeparticle Schrödinger operators in $\mathbb{R}^{3}$, there is the remarkable phenomenon known as Efimov effect: if all Hamiltonians of the two-body subsystems are nonnegative and if at least two of them have a zero-energy resonance, then the threebody system has an infinite number of negative eigenvalues accumulating at zero.

This remarkable spectral property was discovered by Efimov [1] and has since become the subject of many papers [2-11]. The first mathematical proof of the existence of this effect was given by Jafaev [11], and Szlachányi and Vecsernyés [8] established the asymptotics for the number of eigenvalues near the threshold of the essential spectrum.

Recently, Wang [12] has proved the existence of the Efimov effect in the system with $N \geq 4$ particles in $\mathbb{R}^{3}$ but in this case the properties of the spectrum have not been fully comprehended yet.

In statistical physics $[13,14]$, solid-state physics $[15,16]$, and the theory of quantum fields [17-19], some important problems arise where the number of quasiparticles is bounded, but not fixed. The authers of [20] have developed geometric and commutator techniques to find the location of the spectrum and to prove absence of singular continuous spectrum for Hamiltonians without conservation of the particle number.

Notice that the study of systems describing $n$ particles in interaction, without conservation of the number of particles, is reduced to the investigation of the spectral properties of self-adjoint operators acting in the cut subspace $\mathscr{H}^{(n)}$ of the Fock space, consisting of $r \leq n$ particles [13, 16-18, 20, 21].

The model operator, associated with a system describing two bosons and one particle, another nature in interaction, 
without conservation of the number of particles, was considered in [22, 23], and the existence of the Efimov effect was proved. This model operator acts in the direct sum of zero-, one-, and two-particle subspaces of the bosonic Fock space $\mathscr{F}_{S}\left(L^{2}\left(\mathbb{T}^{3}\right)\right)$ over $L^{2}\left(\mathbb{T}^{3}\right)$.

In the present paper, we consider a model operator $H$, acting in the direct sum of zero-, one-, and two-particle subspaces of the fermionic Fock space $\mathscr{F}_{a}\left(L^{2}\left(\mathbb{T}^{3}\right)\right)$ over $L^{2}\left(\mathbb{T}^{3}\right)$, associated with a system describing two identical fermions and one particle, another nature in interactions, without conservation of the number of particles on the threedimensional lattice.

The main aim of the present paper is to study spectral properties for a model operator $H$ with emphasis on the asymptotics for the number of infinitely many eigenvalues (Efimov's effect case).

We admit a general form for the kinetic part of the hamiltonian $H$, which contains a parameter $\gamma$ to distinguish the two identical particles from the third one (this parameter would be the ratio of the mass). Under some smoothness assumptions, we obtain the following results.

(i) We find a critical value $\gamma^{*}$ for the parameter $\gamma$ that allows or forbids the Efimov effect and we prove that only for $\gamma<\gamma^{*}$ the Efimov effect is absent, while this effect exists for any $\gamma>\gamma^{*}$.

(ii) In the case $\gamma>\gamma^{*}$, we also establish the following asymptotics for the number $N(z)$ of eigenvalues below $z<E_{\text {min }}$ :

$$
\lim _{z \rightarrow E_{\min }^{-}} \frac{N(z)}{|\log | E_{\min }-z||}=\mathscr{U}_{0}(\gamma) \quad\left(\mathscr{U}_{0}(\gamma)>0\right), \forall \gamma>\gamma^{*} \text {. }
$$

We notice that the assertion (i) is surprising and similar assertions are not true for the lattice model operators in the bosonic Fock space $[22,23]$, but in both cases, the presence of the Efimov effect is due to the annihilation and creation operators.

However, the presence of the Efimov effect for the threeparticle Schrödinger operators is due to the two-particle interaction operators (see, e.g., $[8-11,24,25]$ in lattice case).

We remark that for a model operator $H$ associated to a system describing three particles on the lattice the authors Dell'Antonio et al. [26] found an explicit value of the parameter $\gamma$, say $\gamma^{*}$, such that only for values of $\gamma$ below this number, the Efimov effect is absent for the sector of the Hilbert space which contains functions which are antisymmetric with respect to the two identical particles, while it is present for all values of the parameter $\gamma$ on the symmetric sector. In case of antisymmetric wavefunction, the number $\gamma^{*}$ is a critical value for the mass ratio, where the Efimov effect is present or absent.

In the continuous case, interestingly the case of a system of three fermions, two identical and different from the third one, with short-range interaction, was also considered from a more physical point of view by Petrov [27], and he also found a critical value for the mass ratio $\approx 13,6$ (in our case $\gamma^{*} \approx 13,60696570$ ) that permits or forbids the Efimov effect.
The organization of the present paper is as follows. Section 1 is an introduction to the whole work. In Section 2, the model operator is described as a bounded self-adjoint operator $H$ in $\mathscr{H}^{(3)}$. Some spectral properties of the corresponding Friedrichs models $h(p), p \in \mathbb{T}^{3}$, are studied, the location and structure of the essential spectrum of $H$ are given, and the main result of the paper is formulated. Section 3 deals with the review the Birman-Schwinger principle for the operator $H$. In Section 4, we prove of the main result.

In order to facilitate a description of the content of this paper, we briefly introduce the notation used throughout this paper. Let $\mathbb{T}^{3}=(\mathbb{R} / 2 \pi \mathbb{Z})^{3}=(-\pi, \pi]^{3}$ be the threedimensional torus (the first Brillouin zone, i.e., the dual group of $\mathbb{Z}^{3}$ ) equipped with its Haar measure. Denote by $L^{2}(\Omega)$ the Hilbert space of square-integrable functions defined on a measurable set $\Omega \subset \mathbb{R}^{n}$. Denote by $L_{\text {as }}^{2}\left(\left(\mathbb{T}^{3}\right)^{2}\right)$ the subspace of antisymmetric functions of the Hilbert space $L^{2}\left(\left(\mathbb{T}^{3}\right)^{2}\right)$. We denote by $c, C$ several constants, whose magnitudes are not of interest.

\section{The Model Operator and Statement of the Main Results}

Set

$$
\mathscr{H}_{0}=\mathbb{C}, \quad \mathscr{H}_{1}=L^{2}\left(\mathbb{T}^{3}\right), \quad \mathscr{H}_{2}=L_{\text {as }}^{2}\left(\left(\mathbb{T}^{3}\right)^{2}\right) .
$$

The Hilbert space $\mathscr{H}^{(3)}=\mathscr{H}_{0} \oplus \mathscr{H}_{1} \oplus \mathscr{H}_{2}$ is called the direct sum of zero-, one-, and two-particle subspaces of a fermionic Fock space $\mathscr{F}_{a}\left(L^{2}\left(\mathbb{T}^{3}\right)\right)$ over $L^{2}\left(\mathbb{T}^{3}\right)$.

Let $H_{i j}$ be annihilation (creation) operators [18] defined in the Fock space for $i<j(i>j)$. We note that in physics, an annihilation operator is an operator that lowers the number of particles in a given state by one, a creation operator is an operator that increases the number of particles in a given state by one, and it is the adjoint of the annihilation operator.

In this paper, we consider the case, where the number of annihilations and creations of the particles of the considering system is equal to 1 . It means that $H_{i j} \equiv 0$ for all $|i-j|>1$. So, the model operator $H$ associated with the energy operator of a system describing three particles in interaction, without conservation of the number of particles, acts in the Hilbert space $\mathscr{H}^{(3)}$ as a matrix operator

$$
H=\left(\begin{array}{ccc}
H_{00} & H_{01} & 0 \\
H_{10} & H_{11} & H_{12} \\
0 & H_{21} & H_{22}
\end{array}\right)
$$

where the operators $H_{i j}: \mathscr{H}_{j} \rightarrow \mathscr{H}_{i}, i, j=0,1,2$ are defined by the forms

$$
\begin{gathered}
\left(H_{00} f_{0}\right)_{0}=u_{0} f_{0}, \quad\left(H_{01} f_{1}\right)_{0}=\int_{\mathbb{T}^{3}} b\left(q^{\prime}\right) f_{1}\left(q^{\prime}\right) d q^{\prime}, \\
\left(H_{10} f_{0}\right)_{1}=b(p) f_{0}, \quad\left(H_{11} f_{1}\right)_{1}=u(p) f_{1}(p), \\
\left(H_{12} f_{2}\right)_{1}=\int_{\mathbb{T}^{3}} b\left(q^{\prime}\right) f_{2}\left(p, q^{\prime}\right) d q^{\prime},
\end{gathered}
$$




$$
\begin{gathered}
\left(H_{21} f_{1}\right)_{2}=\frac{1}{2}\left(b(q) f_{1}(p)-b(p) f_{1}(q)\right), \\
\left(H_{22} f_{2}\right)_{2}=E(p, q) f_{2}(p, q) .
\end{gathered}
$$

Here $f_{i} \in \mathscr{H}_{i}, i=0,1,2, u_{0}$-fixed real number, $b(\cdot), u(\cdot)$ real-valued analytic functions on $\mathbb{T}^{3}$ and $E(\cdot, \cdot)$ is a realanalytic symmetric function defined on $\left(\mathbb{T}^{3}\right)^{2}$.

Under these assumptions, the operator $H$ is bounded and self-adjoint in $\mathscr{H}^{(3)}$.

Throughout this paper we assume the following additional technical assumptions.

Hypothesis 1. (a) The real-analytic function $E(\cdot, \cdot)$ which is symmetric on $\left(\mathbb{T}^{3}\right)^{2}$ is even with respect to $(p, q)$ and has a unique nondegenerate zero minimum at the origin $(0,0) \epsilon$ $\left(\mathbb{T}^{3}\right)^{2}$ and there exist positive definite matrix $W$ and positive real number $\gamma>0$ such that

$$
\begin{gathered}
\left(\frac{\partial^{2} E(0,0)}{\partial p_{i} \partial p_{j}}\right)_{i, j=1}^{3}=(1+\gamma) W, \\
\left(\frac{\partial^{2} E(0,0)}{\partial p_{i} \partial q_{j}}\right)_{i, j=1}^{3}=\gamma W .
\end{gathered}
$$

(b) The functions $u(\cdot)$ and $b(\cdot)$ on $\mathbb{T}^{3}$ are even and $u(\cdot)$ has a unique minimum at the origin.

By Hypothesis 1 , for any $p \in \mathbb{T}^{3}$ the integral

$$
\int_{\mathbb{T}^{3}} \frac{b^{2}(t) d t}{E(p, t)-E_{\min }}
$$

is finite and hence it defines a continuous even function on $\mathbb{T}^{3}$, which will be denoted by $\Lambda(p)$.

Since the function $E(p, q)$ has a unique nondegenerate minimum at the point $(0,0) \in\left(\mathbb{T}^{3}\right)^{2}$ and $E(0,0)=E_{\min }$ the function $\Lambda(p)$ is positive.

Hypothesis 2. For any nonzero $p \in \mathbb{T}^{3}$, the inequality $\Lambda(p)<$ $\Lambda(0)$ holds.

Remark 1. For the functions

$$
\begin{gathered}
u(p)=\varepsilon(p)+c, \quad b(p)=\varepsilon(p), \\
E(p, q)=\gamma \varepsilon(p+q)+\varepsilon(p)+\varepsilon(q), \quad p, q \in \mathbb{T}^{3},
\end{gathered}
$$

where $c>0$ is a real number and $\varepsilon(\cdot)$ is real valued analytic conditionally negative definite function with a unique minimum at the origin, Hypotheses 1 and 2 are fulfilled (see [22, Lemma A1]).

Recall that a complex-valued bounded function $\varepsilon: \mathbb{T}^{m} \rightarrow$ $\mathbb{C}$ is called conditionally negative definite if $\varepsilon(p)=\overline{\varepsilon(-p)}$ and

$$
\sum_{i, j=1}^{n} \varepsilon\left(p_{i}-p_{j}\right) z_{i} \bar{z}_{j} \leq 0
$$

for all $p_{1}, \ldots, p_{n} \in \mathbb{T}^{m}$ and all $\mathbf{z}=\left(z_{1}, \ldots, z_{n}\right) \in \mathbb{C}^{n}$ satisfying $\sum_{i=1}^{n} z_{i}=0$.

2.1. The Friedrichs Model. To formulate the main results of the paper we introduce a family of Friedrichs models $h(p)$, $p \in \mathbb{T}^{3}$, which act in $\mathscr{H}^{(2)} \equiv \mathscr{H}_{0} \oplus \mathscr{H}_{1}$ with the entries

$$
\begin{gathered}
\left(h_{00}(p) f_{0}\right)_{0}=u(p) f_{0}, \quad h_{01}=\frac{1}{\sqrt{2}} H_{01}, \\
h_{10}=h_{01}^{*}, \quad\left(h_{11}(p) f_{1}\right)_{1}(q)=\mathfrak{e}_{p}(q) f_{1}(q),
\end{gathered}
$$

where $\boldsymbol{e}_{p}(\cdot)=E(p, \cdot)$.

Let the operator $h_{0}(p), p \in \mathbb{T}^{3}$, act in $\mathscr{H}^{(2)}$ as

$$
h_{0}(p)\left(\begin{array}{c}
f_{0} \\
f_{1}(q)
\end{array}\right)=\left(\begin{array}{c}
0 \\
\boldsymbol{e}_{p}(q) f_{1}(q)
\end{array}\right) .
$$

The perturbation $h(p)-h_{0}(p)$ of the operator $h_{0}(p)$ is a self-adjoint operator of rank 2 . Therefore, in accordance with the invariance of the essential spectrum under finite rank perturbations, the essential spectrum $\sigma_{\text {ess }}(h(p))$ of $h(p)$ fills the following interval on the real axis:

$$
\sigma_{\text {ess }}(h(p))=[m(p), M(p)],
$$

where

$$
m(p)=\min _{q \in \mathbb{T}^{3}} \mathfrak{e}_{p}(q), \quad M(p)=\max _{q \in \mathbb{T}^{3}} \mathfrak{e}_{p}(q) .
$$

Definition 2. Let $u(0) \neq E_{\min }$. The operator $h(0)$ is said to have a threshold resonance if the number 1 is an eigenvalue of the operator

$$
(\mathrm{G} \psi)(q)=\frac{b(q)}{2\left(u(0)-E_{\min }\right)} \int_{\mathbb{T}^{3}} \frac{b(t) \psi(t) d t}{\mathfrak{e}_{0}(t)-E_{\min }}, \quad \psi \in C\left(\mathbb{T}^{3}\right),
$$

and the associated eigenfunction $\psi$ (up to a constant factor) satisfies the condition $\psi(0) \neq 0$.

Remark 3. The spectrum and resonances of this Friedrichs model are studied in $[22,23]$.

For any $p \in \mathbb{T}^{3}$, we define an analytic function $\Delta(p, z)$ (the Fredholm determinant associated with the operator $h(p))$ in $\mathbb{C} \backslash[m(p), M(p)]$ by

$$
\Delta(p, z)=u(p)-z-\frac{1}{2} \int_{\mathbb{T}^{3}} \frac{b^{2}(t) d t}{E(p, t)-z} .
$$

Since $\Delta(0, \cdot)$ is continuous in $z \leq E_{\min }$, the following finite limit exists:

$$
\Delta\left(0, E_{\min }\right)=\lim _{z \rightarrow E_{\min }} \Delta(0, z)
$$

Lemma 4. For any $p \in \mathbb{T}^{3}$ the operator $h(p)$ has an eigenvalue $z \in \mathbb{C} \backslash[m(p), M(p)]$ if and only if $\Delta(p, z)=0$. 
Proof. One can see that the equation

$$
h(p) f=z f, \quad f \in \mathscr{H}^{(2)}, z \in \mathbb{C} \backslash[m(p), M(p)],
$$

is equivalent to the following equation:

$$
\begin{aligned}
& \left(h_{00}(p)-z\right) f_{0}+h_{01} f_{1}=0, \\
& f_{1}=-\left(h_{11}(p)-z\right)^{-1} h_{10} f_{0} .
\end{aligned}
$$

Putting $f_{1}$ to the first equation and then according to the equality $\Delta(p, z)=h_{00}(p)-z-h_{01}\left(h_{11}(p)-z\right)^{-1} h_{10}$ we have the fact that (17) is equivalent to the equation

$$
\Delta(p, z) f_{0}=0 .
$$

Thus (17) has nontrivial solution if and only if $\Delta(p, z)=0$.

Lemma 5. (i) The operator $h(0)$ has a threshold resonance if and only if $\Delta\left(0, E_{\min }\right)=0$ and $b(0) \neq 0$.

(ii) If $u(0) \leq E_{\min }$, then the operator $h(0)$ has no a threshold resonance.

(iii) Assume $\Delta\left(0, E_{\min }\right)=0$.

(a) If $b(0) \neq 0$, then the operator $h(0)$ has a threshold resonance and the vector $f=\left(f_{0}, f_{1}\right)$, where

$$
\begin{gathered}
f_{0}=\text { const } \neq 0, \\
f_{1}(q)=-\frac{b(q) f_{0}}{\sqrt{2}\left(\mathfrak{e}_{0}(q)-E_{\min }\right)} \in L^{1}\left(\mathbb{T}^{3}\right) \backslash L^{2}\left(\mathbb{T}^{3}\right),
\end{gathered}
$$

obeys the equation $h(0) f=E_{\min } f$.

(b) If $b(0)=0$, then the number $z=E_{\text {min }}$ is an eigenvalue of the operator $h(0)$ and the vector $f=\left(f_{0}, f_{1}\right)$, where $f_{0} \in \mathbb{C}^{1}$ and $f_{1} \in L^{2}\left(\mathbb{T}^{3}\right)$ defined by (20), is the corresponding eigenvector.

Proof. (i) "Only If Part." Suppose that the operator $h(0)$ has a threshold energy resonance. Then by Definition 2 the number 1 is an eigenvalue of the operator (14) as

$$
\begin{array}{r}
(\mathrm{G} \psi)(q)=\frac{b(q)}{2\left(u(0)-E_{\min }\right)} \int_{\mathbb{T}^{3}} \frac{b(t) \psi(t) d t}{\mathfrak{e}_{0}(t)-E_{\min }}, \\
\psi \in C\left(\mathbb{T}^{3}\right),
\end{array}
$$

and the associated eigenfunction $\psi$ satisfies $\psi(0) \neq 0$.

This solution is equal to the function $b(\cdot)$ (up to a constant factor) and hence $\Delta\left(0, E_{\min }\right)=0$.

"If Part." Let the equality $\Delta\left(0, E_{\text {min }}\right)=0$ hold and let $b(0) \neq 0$. Then the inequality $u(0) \neq E_{\text {min }}$ holds and the function $b(\cdot)$ is an eigenfunction of $G$; that is, by Definition 2 the operator $h(0)$ has a threshold energy resonance.

(ii) Since $u(0) \leq E_{\min }$ and $-h_{01}\left(h_{11}(0)-E_{\min }\right)^{-1} h_{10}<0$, we get $\Delta\left(0, E_{\min }\right)<0$ which ends the proof together with (ii). (iii) Similarly to the proof of Lemma 4 , one can check that $\Delta\left(0, E_{\min }\right)=h_{00}(0)-E_{\min }-h_{01}\left(h_{11}(0)-E_{\min }\right)^{-1} h_{10}$ holds, and it is a determinant of the equation

$$
\begin{gathered}
\left(h_{00}(p)-E_{\min }\right) f_{0}+h_{01} f_{1}=0, \\
f_{1}=-\left(h_{11}(0)-E_{\min }\right)^{-1} h_{10} f_{0}, \\
\text { that is } f_{1}(q)=-\frac{b(q) f_{0}}{\sqrt{2}}\left(e_{0}(q)-E_{\min }\right)^{-1},
\end{gathered}
$$

which is equivalent to the equation

$$
h(0) f=E_{\min } f, \quad f \in \mathbb{C} \oplus L^{1}\left(\mathbb{T}^{3}\right) .
$$

Thus (23) has nontrivial solution if and only if $\Delta(0$, $\left.E_{\text {min }}\right)=0$.

By Hypothesis 1 there exist $c, C>0$ and a bounded, partially continuous $\bar{b}(\cdot)$ function such that

$$
\begin{gathered}
c|q|^{2} \leq \mathfrak{e}_{0}(q)-E_{\min } \leq C|q|^{2}, \\
b(q)=b(0)+\bar{b}(q)|q|^{2} .
\end{gathered}
$$

(a) If $b(0) \neq 0$ by (i), the operator $h(0)$ has a threshold resonance. Due to the fact $1 /|q|^{2} \in L^{1}\left(\mathbb{T}^{3}\right) \backslash L^{2}\left(\mathbb{T}^{3}\right)$ and (24), we have $f_{1} \in L^{1}\left(\mathbb{T}^{3}\right) \backslash L^{2}\left(\mathbb{T}^{3}\right)$.

(b) If $b(0)=0$, then using (24) we can see $f_{1} \in L^{2}\left(\mathbb{T}^{3}\right)$ and $f=\left(f_{0}, f_{1}\right) \in \mathscr{H}^{(2)}$, with $f$ eigenfunction of $h(0)$ corresponding to the eigenvalue $E_{\min }$.

Let $W$ be the $3 \times 3$ matrix defined in Hypothesis 1 and $U_{\delta}(0)=\left\{p \in \mathbb{T}^{3}:|p|<\delta\right\}$ stands for a $\delta(\delta>0)$ neighborhood of the origin. The following Lemma 6 plays a crucial role in the proof of the infiniteness (finiteness, resp.) of the number of eigenvalues lying below the bottom of the essential spectrum for a model operator $H$. The proof may be handled in much the same way as in $[22,23]$.

Lemma 6. Let the operator $h(0)$ have a threshold resonance. Then for any $p \in U_{\delta}(0), \delta>0$ sufficiently small and $z \leq E_{\min }$, the following decomposition

$$
\begin{aligned}
\Delta(p, z)= & \frac{4 \sqrt{2} \pi^{2} b^{2}(0)}{(1+\gamma)^{3 / 2} \operatorname{det}(W)^{1 / 2}} \sqrt{m(p)-z} \\
& +\Delta^{(02)}(m(p)-z)+\Delta^{(20)}(p, z)
\end{aligned}
$$

holds, where $\Delta^{(02)}(m(p)-z)\left(\Delta^{(20)}(p, z)\right.$, resp. $)$ is a function behaving like $O(m(p)-z)\left(O\left(|p|^{2}\right)\right.$, resp. $)$ as $|m(p)-z| \rightarrow 0$ ( $p \rightarrow 0$ uniformly in $z \leq E_{\min }$, resp.).

Lemma 7. Let the operator $h(0)$ have a threshold resonance. Then there exist positive numbers $c, C$ and $\delta$ such that

$$
\begin{gathered}
c|p| \leq \Delta\left(p, E_{\min }\right) \leq C|p| \quad \text { for any } p \in U_{\delta}(0), \\
\Delta\left(p, E_{\min }\right) \geq c \quad \text { for any } p \in \mathbb{T}^{3} \backslash U_{\delta}(0) .
\end{gathered}
$$


Proof. By the assumption (a) of Hypothesis 1, we have

$$
E(p, q)=E_{\min }+F(p, q)+O\left(|p|^{4}+|q|^{4}\right) \quad \text { as } p, q \longrightarrow 0
$$

where

$$
\begin{aligned}
& F(p, q) \\
& \quad=\frac{1}{2}((1+\gamma)(W p, p)+2 \gamma(W p, q)+(1+\gamma)(W q, q)) .
\end{aligned}
$$

Since $E(\cdot, \cdot)$ has a nondegenerate minimum at the origin and the equality

$$
\min _{q \in \mathbb{T}^{3}} F(p, q)=\frac{1}{2} \frac{1+2 \gamma}{1+\gamma}(W p, p)
$$

Holds, we have

$$
\begin{aligned}
m(p) & =\min _{q \in \mathbb{T}^{3}} E(p, q) \\
& =E_{\min }+\frac{1}{2} \frac{1+2 \gamma}{1+\gamma}(W p, p)+O\left(|p|^{4}\right) \quad \text { as } p \longrightarrow 0
\end{aligned}
$$

Consequently, these asymptotics and Lemma 6 yield (26) for some positive numbers $c, C$.

By Hypotheses 1 and 2 , we have $u(p)>u(0)$ and $\Lambda(p)>$ $\Lambda(0), 0 \neq p \in \mathbb{T}^{3}$, and hence

$$
\begin{aligned}
& \Delta\left(p, E_{\min }\right) \\
& \quad=u(p)-E_{\min }-\frac{1}{2} \Lambda(p)>\Delta\left(0, E_{\min }\right), \quad 0 \neq p \in \mathbb{T}^{3} .
\end{aligned}
$$

Then the inequality (27) follows from $\Delta\left(0, E_{\text {min }}\right)=0$ and the continuity of the function $\Delta\left(p, E_{\min }\right)$.

2.2. The Essential Spectrum of $H$. The following theorem describes the essential spectrum of the operator $H$. Similar results were obtained in the bosonic Fock space in $[22,23]$, and we refer to these paper for the proof.

Theorem 8. For the essential spectrum $\sigma_{\text {ess }}(H)$ of the operator $H$, the equality

$$
\sigma_{\text {ess }}(H)=\bigcup_{p \in \mathbb{T}^{3}} \sigma_{d}(h(p)) \cup\left[E_{\min }, E_{\max }\right]
$$

holds, where $\sigma_{d}(h(p))$ is the discrete spectrum of the operator $h(p), p \in \mathbb{T}^{3}$.

The following lemma describes the location of the essential spectrum of the operator $H$.

Lemma 9. Let Hypothesis 1 be fulfilled and $h(0)$ has a threshold resonance. Then

$$
\sigma_{\text {ess }}(H)=\left[E_{\text {min }}, E_{\text {max }}\right] \cup \sigma_{2},
$$

where $\sigma_{2}=\left\{z \in\left[E_{\max },+\infty\right): \Delta(p, z)=0\right.$ for some $\left.p \in \mathbb{T}^{3}\right\}$.
Proof. According to Lemma 4, the zero of $\Delta(p, \cdot)$ is the eigenvalues of $h(p)$, so

$$
\begin{aligned}
\Upsilon & =\bigcup_{p \in \mathbb{T}^{3}} \sigma_{d}(h(p)) \backslash\left(E_{\min }, E_{\max }\right) \\
& =\left\{z \in \mathbb{R} \backslash\left[E_{\min }, E_{\max }\right]: \Delta(p, z)=0 \text { for some } p \in \mathbb{T}^{3}\right\} .
\end{aligned}
$$

By analyticity of $\Delta(p, \cdot)$, the subset $\Upsilon$ of the essential spectrum of $\sigma_{\text {ess }}(H)$ is described as a finite union of nonintersecting closed intervals.

The function $\Delta(p, \cdot)$ is decreasing in $\left(-\infty, E_{\min }\right)$, respectively, with $\left(E_{\max },+\infty\right)$, and hence there may exist its unique zero lying below, respectively, above the closed interval $\left[E_{\min }, E_{\max }\right]$.

So, the set $\Upsilon$ may be described as the union of intervals $\sigma_{1}$ and $\sigma_{2}$ lying below and above of $\left[E_{\min }, E_{\max }\right]$, and

$$
\begin{aligned}
& \sigma_{1}=\left\{z \in\left(-\infty, E_{\min }\right]: \Delta(p, z)=0 \text { for some } p \in \mathbb{T}^{3}\right\}, \\
& \sigma_{2}=\left\{z \in\left[E_{\max },+\infty\right): \Delta(p, z)=0 \text { for some } p \in \mathbb{T}^{3}\right\} .
\end{aligned}
$$

By virtue of Hypothesis 2 , we have $\Delta\left(p, E_{\min }\right) \geq \Delta(0$, $\left.E_{\text {min }}\right)$ and using Lemma 5 we get $\Delta\left(0, E_{\text {min }}\right)=0$, and so $\Delta(p, z)>\Delta\left(p, E_{\min }\right) \geq \Delta\left(p, E_{\min }\right)=0$, that is

$$
\Delta(p, z)>0 \quad \forall z<E_{\min }
$$

since $\Delta(p, \cdot)$ is decreasing in $\left(-\infty, E_{\min }\right)$. Then according to Lemma 4 we get $\sigma_{1}=\emptyset$.

Consequently, $\cup_{p \in \mathbb{T}^{3}} \sigma_{d}(h(p)) \backslash\left(E_{\min }, E_{\max }\right)=\sigma_{2}$ and Theorem 8 ends the proof of the lemma.

2.3. Statement of the Main Results. Henceforth we assume that $h(0)$ has a threshold resonance.

According to Lemma 9 for the bottom of the essential spectrum the equality $E_{\min }=\inf \sigma_{\text {ess }}(H)$ is valid, and by $N(z)$ we denote the number of eigenvalues and counted according to their multiplicities of $H$ lying below $z \leq E_{\min }$.

Let $\gamma^{*}$ be a solution of the equation

$$
\frac{2(1+\gamma)^{2}}{\pi \gamma \sqrt{1+2 \gamma}}-\frac{2(1+\gamma)^{2}}{\pi \gamma^{2}} \arcsin \frac{\gamma}{1+\gamma}=1, \quad \gamma>0 .
$$

Since the function at the r.h.s. of (38) is continuous, strictly increasing and surjective from $(0, \infty)$ to $(0, \infty)$, the number $\gamma^{*}$ is a unique positive solution.

In the following main theorem, we aprecisely describe the dependence of the number of eigenvalues of $H$ on the parameters $\gamma>0$.

Theorem 10. Let Hypothesis 1 be fulfilled and let $h(0)$ have a threshold resonance at $E_{\mathrm{min}}$. Then we have the following.

(i) For any $0<\gamma<\gamma^{*}$, the operator $H$ has a finite number of eigenvalues lying below the bottom $E_{\min }$ of the essential spectrum. 
(ii) For any $\gamma>\gamma^{*}$, the operator $H$ has infinitely many eigenvalues lying below $E_{\min }$. The function $N(z)$ obeys the relation

$$
\lim _{z \rightarrow E_{\min }^{-}} \frac{N(z)}{|\log | E_{\min }-z||}=\mathscr{U}_{0}(\gamma) \quad\left(\mathscr{U}_{0}(\gamma)>0\right) \text {. }
$$

It follows from the positivity of the limit (39) that the discrete spectrum of the operator $H$ is infinite.

Remark 11. The constant $\mathscr{U}_{0}(\gamma)$ is given as a positive function depending only on the variable $\gamma, \gamma>\gamma^{*}$.

Remark 12. In [26], a result analogue to Theorem 10 has been proven for a model operator $H$ associated to a system describing three particles on the lattice $\mathbb{Z}^{d}$.

\section{The Birman-Schwinger Principle}

For a bounded self-adjoint operator $B$, we define $n(\lambda, B)$ by

$$
n(\lambda, B)=\sup \{\operatorname{dim} F:(B u, u)>\lambda, u \in F,\|u\|=1\}
$$

$n(\lambda, B)$ is equal to infinity if $\lambda$ is in the essential spectrum of $B$ and if $n(\lambda, B)$ is finite, it is equal to the number of the eigenvalues of $B$ larger than $\lambda$. By the definition of $N(z)$, we have

$$
N(z)=n(-z,-H), \quad-z>-E_{\min } .
$$

In our analysis of the spectrum of $H$, the crucial role is played by the self-adjoint compact Faddeev-Newton-type integral operator $T(z), z<E_{\text {min }}$, in the space $\mathscr{H}^{(2)}$ with the entries

$$
\begin{gathered}
\left(T_{00}(z) f_{0}\right)_{0}=\left(1-u_{0}-z\right) f_{0}, \\
\left(T_{01}(z) f_{1}\right)_{0}=-\int_{\mathbb{T}^{3}} \frac{b\left(q^{\prime}\right) f_{1}\left(q^{\prime}\right) d q^{\prime}}{\sqrt{\Delta\left(q^{\prime}, z\right)}}, \\
T_{10}(z)=T_{01}^{*}(z), \\
\left(T_{11}(z) f_{1}\right)_{1}(p) \\
=-\frac{b(p)}{2 \sqrt{\Delta(p, z)}} \int_{\mathbb{T}^{3}} \frac{b\left(q^{\prime}\right) f_{1}\left(q^{\prime}\right) d q^{\prime}}{\sqrt{\Delta\left(q^{\prime}, z\right)}\left(E\left(p, q^{\prime}\right)-z\right)} . \\
\text { Now we describe } T(z) \text { with the entries of } H \text { in }(3) . \text { We } \\
\text { split } H_{21} \text { into the sum of two operators } \\
H_{21}=\frac{1}{2}\left(H_{21}^{(1)}-H_{21}^{(2)}\right), \quad\left(H_{21}^{(1)} f_{1}\right)_{2}=b(q) f_{1}(p), \\
\left(H_{21}^{(2)} f_{1}\right)_{2}=b(p) f_{1}(q),
\end{gathered}
$$

where $f_{1} \in \mathscr{H}_{1}$.
According to the last representation and (3), we take

$$
\begin{gathered}
T_{00}(z)=(1+z) I_{0}-H_{00}, \quad T_{01}(z)=-H_{01} D^{-1 / 2}(z), \\
T_{10}(z)=-D^{-1 / 2}(z) H_{10} \\
T_{11}(z)=-\frac{1}{2} D^{-1 / 2}(z) H_{12} R_{22}^{1 / 2}(z) H_{21}^{(2)} D^{-1 / 2}(z)
\end{gathered}
$$

where $R_{22}(z)=\left(H_{22}-z I_{2}\right)^{-1}$ is the resolvent of $H_{22}$,

$$
D(z)=H_{11}-z I_{1}-\frac{1}{2} D^{-1 / 2}(z) H_{12} R_{22}^{1 / 2}(z) H_{21}^{(1)} D^{-1 / 2}(z) .
$$

One can verify that $D(z)$ is the multiplication operator by the determinant $\Delta(\cdot, z)$. So, logically we may denote by $D^{-1 / 2}(z)$ the multiplication operator by $\Delta^{-1 / 2}(\cdot, z)$.

The following lemma is a modification of the BirmanSchwinger principle for the three-particle Schrödinger operators (see $[8,10,24])$.

Lemma 13. The operator $T(z)$ is compact and continuous in $z<E_{\min }$ and

$$
N(z)=n(1, T(z)) .
$$

Proof. Set

$$
M(z)=\mathscr{R}^{1 / 2}(z)\left(\mathscr{R}^{-1}(z)-(H-z I)\right) \mathscr{R}^{1 / 2}(z),
$$

where $\mathscr{R}(z)=\operatorname{diag}\left\{I_{0}, I_{1}, \mathscr{R}_{22}(z)\right\}$ and $\mathscr{R}^{-1}(z)$ is the inverse of $\mathscr{R}(z)$.

For entries of matrix operator $M(z)$, we have

$$
\begin{gathered}
M_{00}(z)=(1+z) I_{0}-H_{00}, \quad M_{01}(z)=-H_{01}, \\
M_{02}(z)=0, \\
M_{10}(z)=-H_{10}, \quad M_{11}(z)=(1+z) I_{1}-H_{11}, \\
M_{12}(z)=-H_{12} R_{22}^{1 / 2}(z), \\
M_{20}(z)=0, \quad M_{21}(z)=-R_{22}^{1 / 2}(z) H_{21}, \\
M_{22}(z)=0 .
\end{gathered}
$$

The correctness of

$$
N(z)=n(1, M(z))
$$

is shown by the fact $g \in \mathscr{H}_{-H}(-z)$, that is, $(H g, g)<z(g, g)$ if and only if $(\hat{g}, \widehat{g})<(M(z) \hat{g}, \widehat{g}), \widehat{g} \in \mathscr{H}_{M(z)}(1)$, where $\widehat{g}=$ $\mathscr{R}^{-1 / 2}(z) g$.

For any $z<\tau_{\text {ess }}(H)$, we define an operator $V(z)$ acting in the Hilbert space $\mathscr{H}_{0} \oplus \mathscr{H}_{1}$ by the form

$$
V(z)=\left(\begin{array}{cc}
M_{00}(z) & M_{01}(z) \\
M_{10}(z) & M_{11}(z)+M_{12}(z) M_{21}(z)
\end{array}\right)
$$


and prove the equality

$$
N(z)=n(1, V(z)), \quad z<\tau_{\mathrm{ess}}(H) .
$$

Let $f=\left(f_{0}, f_{1}\right) \in \mathscr{H}_{V(z)}(1)$, and then for $g=\left(f_{0}, f_{1}\right.$, $\left.-M_{21}(z) f_{1}\right) \in \mathscr{H}^{(3)}$, we get

$$
\begin{aligned}
(M(z) g, g) & =(V(z) f, f)+\left(M_{21}(z) f_{1}, g_{2}\right) \\
& >(f, f)+\left(g_{2}, g_{2}\right)=(g, g),
\end{aligned}
$$

that is, $g \in \mathscr{H}_{M(z)}(1)$.

Therefore,

$$
n(1, V(z)) \leq n(1, M(z)), \quad z<\tau_{\text {ess }}(H) .
$$

Using the equality

$$
\begin{aligned}
(V(z) f, f)= & (M(z) g, g)+\left(M_{12}(z) M_{21}(z) g_{1}, g_{1}\right) \\
& -\left(M_{21}(z) g_{1}, g_{2}\right)-\left(M_{12}(z) g_{2}, g_{1}\right),
\end{aligned}
$$

where $g \in \mathscr{H}^{(3)}, f=\left(g_{1}, g_{2}\right) \in \mathscr{H}^{(2)}$, and taking $g=\left(g_{0}, g_{1}\right.$, $\left.g_{2}\right) \in \mathscr{H}_{M(z)}(1)$ realized analogously, we get

$$
\begin{array}{r}
(V(z) f, f)>(f, f)+\left\|g_{2}-M_{21}(z) g_{1}\right\|^{2} \geq(f, f), \\
\text { that is, } f \in \mathscr{H}_{V(z)}(1)
\end{array}
$$

and so

$$
n(1, V(z)) \geq n(1, M(z)), \quad z<\tau_{\text {ess }}(H) .
$$

Observe that

$$
V(z)-I=\mathscr{D}^{1 / 2}(z)(T(z)-I) \mathscr{D}^{1 / 2}(z),
$$

where $\mathscr{D}^{1 / 2}(z)$ is acting in the Hilbert space $\mathscr{H}_{0} \oplus \mathscr{H}_{1}$ by the equality

$$
\mathscr{D}^{1 / 2}(z)=\operatorname{diag}\left\{I_{0}, \mathscr{D}^{1 / 2}(z)\right\} \text {. }
$$

Employing (57), we show $g \in \mathscr{H}_{V(z)}(1)$, that is, $(V(z) g, g)>(g, g)$ if and only if $(T(z) f, f)>(f, f), f=$ $\mathscr{D}^{-1 / 2}(z) g \in \mathscr{H}_{T(z)}(1)$
Consequently, for any $z<\tau_{\text {ess }}(H)$, we have $n(1, V(z))=n(1, T(z)), \quad$ that is $N(z)=n(1, T(z))$.

3.1. The Birman-Schwinger Principle at the Threshold. It should be noted that the operator $T(z)$ can be defined as a bounded operator even for the point $z=E_{\min }$ by

$$
\begin{gathered}
\left(T_{00}\left(E_{\min }\right) f_{0}\right)_{0}=\left(1-u_{0}-E_{\min }\right) f_{0}, \\
\left(T_{01}\left(E_{\min }\right) f_{1}\right)_{0}=-\int_{\mathbb{T}^{3}} \frac{b\left(q^{\prime}\right) f_{1}\left(q^{\prime}\right) d q^{\prime}}{\sqrt{\Delta\left(q^{\prime}, z\right)}},
\end{gathered}
$$

$$
T_{10}\left(E_{\min }\right)=T_{01}^{*}\left(E_{\min }\right),
$$

$$
\begin{aligned}
& \left(T_{11}\left(E_{\min }\right) f_{1}\right)_{1}(p) \\
& \quad=-\frac{b(p)}{2 \sqrt{\Delta\left(p, E_{\min }\right)}} \int_{\mathbb{T}^{3}} \frac{b\left(q^{\prime}\right) f_{1}\left(q^{\prime}\right) d q^{\prime}}{\sqrt{\Delta\left(q^{\prime}, E_{\min }\right)}\left(E\left(p, q^{\prime}\right)-E_{\min }\right)} .
\end{aligned}
$$

Remark 14. The operator $T(z)$ converges strongly (but not uniformly) as $z \rightarrow E_{\min }-0$ to $T\left(E_{\min }\right)$. Here we do not give the proof of this convergence. The convergence of the these types of operators was shown in $[11,25]$.

The next lemma can be proved analogously as Lemma 13.

Lemma 15. For any $z, z \leq E_{\min }$, the inequality

$$
N(z) \leq n\left(1, T\left(E_{\min }\right)\right)
$$

occurs.

\section{The Sketch of the Proof of the Main Results}

By Hypothesis 1, we get

$$
\begin{aligned}
E(p, q)= & E_{\min }+\frac{1}{2}((1+\gamma)(W p, p) \\
& +2 \gamma(W p, q)+(1+\gamma)(W q, q)) \\
& +O\left(|p|^{4}+|q|^{4}\right)
\end{aligned}
$$

as $p, q \rightarrow 0$ and (31) as

$$
m(k)=E_{\min }+\frac{1}{2} \frac{1+2 \gamma}{1+\gamma}(W k, k)+O\left(|k|^{4}\right) \quad \text { as } k \longrightarrow 0 .
$$

Applying the asymptotics for $m(p)$ and using Lemma 6, we have

$$
\begin{aligned}
& \Delta(p, z) \\
& =\frac{4 \pi^{2} b^{2}(0)}{(1+\gamma)^{3 / 2} \operatorname{det}(W)^{1 / 2}}\left[n(W p, p)+2\left(E_{\min }-z\right)\right]^{1 / 2} \\
& \quad+O\left(|p|^{2}+\left|E_{\min }-z\right|\right) \quad \text { as } p,\left|E_{\min }-z\right| \longrightarrow 0
\end{aligned}
$$


where

$$
n=\frac{1+2 \gamma}{1+\gamma}
$$

4.1. The Infiniteness of the Discrete Spectrum of H. In this subsection we will derive the asymptotics (39) for the number of eigenvalues of $H$.

We recall that in this subsection we closely follow Sobolev's method (see [8]) to derive the asymptotics for the number of eigenvalues of the operator $H$ (see Theorem 10).

Let $T\left(\delta ;\left|z-E_{\min }\right|\right)$ be the operator in $\mathscr{H}^{(2)}$ defined by

$$
T\left(\delta ;\left|z-E_{\min }\right|\right)=\left(\begin{array}{lc}
0 & 0 \\
0 & T_{11}\left(\delta ;\left|z-E_{\min }\right|\right)
\end{array}\right),
$$

where the $T_{11}\left(\delta ;\left|E_{\min }-z\right|\right)$ is the integral operator in $\mathscr{H}_{1}$ with the kernel

$$
\begin{aligned}
T\left(\delta, \mid E_{\min }-\right. & z \mid ; p, q) \\
=-d_{0}(( & \widehat{\chi}_{\delta}(p) \hat{\chi}_{\delta}(q)\left(n(W p, p)+2\left|E_{\min }-z\right|\right)^{-1 / 4} \\
& \left.\times\left(n(W q, q)+2\left|E_{\min }-z\right|\right)^{-1 / 4}\right) \\
& \times((1+\gamma)(W p, p)+2 \gamma(W p, q) \\
& \left.\left.+(1+\gamma)(W q, q)+2\left|E_{\min }-z\right|\right)^{-1}\right),
\end{aligned}
$$

where $\widehat{\chi}_{\delta}(\cdot)$ is the characteristic function of the region $\widehat{U}_{\delta}(0)=$ $\left\{p \in \mathbb{T}^{3}:\left|W^{1 / 2} p\right|<\delta\right\}$ and

$$
d_{0}=\frac{\operatorname{det} W^{1 / 2}}{2 \pi^{2}}(1+\gamma)^{3 / 2} .
$$

Lemma 16. Let the conditions of Theorem 10 be fulfilled. The operator $T(z)-T\left(\delta ;\left|E_{\min }-z\right|\right)$ belongs to the Hilbert-Schmidt class and is continuous in $z \leq E_{\min }$.

Proof. Applying asymptotics (62) and (64), one can estimate the kernel of the operator $T(z)-T\left(\delta ;\left|E_{\min }-z\right|\right), z \leq E_{\min }$, by the square-integrable function

$$
\begin{aligned}
& C\left(\frac{|p|^{2}+|q|^{2}}{|p|^{1 / 2}\left(p^{2}+q^{2}\right)|q|^{1 / 2}}\right. \\
& \left.\quad+\frac{\left|E_{\min }-z\right|^{1 / 2}\left(p^{2}+q^{2}\right)^{-1}}{\left(|p|^{2}+\left|E_{\min }-z\right|\right)^{1 / 4}\left(|q|^{2}+\left|E_{\min }-z\right|\right)^{1 / 4}}+1\right) .
\end{aligned}
$$

Hence the operator $T(z)-T\left(\delta ;\left|E_{\min }-z\right|\right)$ belongs to the Hilbert-Schmidt class for all $z \leq E_{\min }$. In combination with the continuity of the kernel of the operator in $z<E_{\min }$, this gives the continuity of $T(z)-T\left(\delta ;\left|E_{\min }-z\right|\right)$ in $z \leq E_{\min }$.
Let $\widehat{T}_{0}\left(\delta ;\left|E_{\min }-z\right|\right)$ be the restriction of the integral operator $T\left(\delta ;\left|E_{\min }-z\right|\right)$ to the subspace $L^{2}\left(\widehat{U}_{\delta}(0)\right)$. One verifies that the operator $\widehat{T}_{0}\left(\delta ;\left|E_{\min }-z\right|\right)$ is unitarily equivalent to the integral operator $T_{1}(r)$ acting in $L^{2}\left(U_{r}(0)\right)$, where $r=$ $\left|E_{\text {min }}-z\right|^{-1 / 2}$ and $U_{r}(0)=\left\{p \in \mathbb{R}^{3}:|p|<r\right\}$, with the kernel

$$
T_{1}(r ; p, q)=-d_{1} \frac{\left(n p^{2}+2\right)^{-1 / 4}\left(n q^{2}+2\right)^{-1 / 4}}{(1+\gamma) p^{2}+2 \gamma(p, q)+(1+\gamma) q^{2}+2},
$$

where

$$
d_{1}=\frac{(1+\gamma)^{3 / 2}}{2 \pi^{2}}
$$

The equivalence is given by the unitary dilation

$$
\begin{gathered}
B: L^{2}\left(\widehat{U}_{\delta}(0)\right) \longrightarrow L^{2}\left(U_{r}(0)\right), \\
\left(B_{r} f\right)(p)=\left(\frac{r}{\delta}\right)^{-3 / 2} f\left(\frac{\delta}{r} W^{1 / 2} p\right) .
\end{gathered}
$$

Furthermore, we may replace

$$
\begin{gathered}
\left(n p^{2}+2\right)^{-1 / 4}, \quad\left(n q^{2}+2\right)^{-1 / 4}, \\
(1+\gamma) p^{2}+2 \gamma(p, q)+(1+\gamma) q^{2}+2
\end{gathered}
$$

by

$$
\begin{gathered}
\left(n p^{2}\right)^{-1 / 4}\left(1-\chi_{1}(p)\right), \quad\left(n q^{2}\right)^{-1 / 4}\left(1-\chi_{1}(q)\right), \\
(1+\gamma) p^{2}+2 \gamma(p, q)+(1+\gamma) q^{2},
\end{gathered}
$$

respectively, since the error will be a Hilbert-Schmidt operator continuous up to $z=E_{\min }$.

We have denoted by $\chi_{1}(\cdot)$ the characteristic function of the ball $U_{1}(0)$. By the replacement, we obtain the integral operator $T_{2}(r)$ in $L^{2}\left(U_{r}(0) \backslash U_{1}(0)\right)$ with a kernel

$$
T_{2}(r ; p, q)=-\frac{d_{1}}{n^{1 / 2}} \frac{|p|^{-1 / 2}|q|^{-1 / 2}}{(1+\gamma) p^{2}+2 \gamma(p, q)+(1+\gamma) q^{2}} .
$$

By the dilation

$$
\begin{gathered}
M: L^{2}\left(U_{r}(0) \backslash U_{1}(0)\right) \longrightarrow L^{2}\left((0, \mathbf{r}), \mathbb{S}^{2}\right), \\
\quad \mathbf{r}=\frac{1}{2}|\log | E_{\min }-z||,
\end{gathered}
$$

where $\mathbb{S}^{2}$ is the unit sphere in $\mathbb{R}^{3},(M f)(x, w)=$ $e^{3 x / 2} f\left(e^{x} w\right), x \in(0, \mathbf{r}), w \in \mathbb{S}^{2}$, one sees that the operator $T_{2}(r)$ is unitarily equivalent to the integral operator $\mathbf{S}_{\mathbf{r}}$ with the kernel $S_{\gamma}\left(x-x^{\prime} ;<\xi, \eta>\right), \xi, \eta \in \mathbb{S}^{2}, x, x^{\prime} \in \mathbb{R}^{+}$, where

$$
\begin{gathered}
S_{\gamma}(x ; t)=-(2 \pi)^{-2} \frac{u}{\cosh x+s t}, \\
u=\frac{1+\gamma}{\sqrt{1+2 \gamma}}, \quad s=\frac{\gamma}{1+\gamma}, \quad t=\langle\xi, \eta\rangle .
\end{gathered}
$$

For the completeness, we reproduce the following lemma, which has been proved in [8]. 
Lemma 17. Let $A(z)=A_{0}(z)+A_{1}(z)$, where $A_{0}\left(A_{1}\right)$ is compact and continuous in $z<0(z \leq 0)$. Assume that for some function $f(\cdot), f(z) \rightarrow 0, z \rightarrow-0$ the limit

$$
\lim _{z \rightarrow-0} f(z) n\left(\lambda, A_{0}(z)\right)=l(\lambda)
$$

exists and is continuous in $\lambda>0$. Then the same limit exists for $A(z)$ and

$$
\lim _{z \rightarrow-0} f(z) n(\lambda, A(z))=l(\lambda) .
$$

The following theorem is important for the proof of the asymptotics (39).

Theorem 18. Let the conditions of the part (ii) of Theorem 10 be fulfilled. The following equalities

$$
\begin{aligned}
\lim _{\left|E_{\min }-z\right| \rightarrow 0} \frac{n\left(1, T_{1}\left(\left|E_{\min }-z\right|^{-1 / 2}\right)\right)}{|\log | E_{\min }-z||} & =\lim _{\mathbf{r} \rightarrow \infty} \frac{1}{2} \mathbf{r}^{-1} n\left(1, \mathbf{S}_{\mathbf{r}}\right) \\
& =\mathscr{U}_{0}(\gamma), \quad \mathscr{U}_{0}(\gamma)>0,
\end{aligned}
$$

hold.

Proof. The coefficient $\mathscr{U}(\gamma)$ in the r.h.s of the asymptotics (80) will be expressed by means of the self-adjoint integral operator $\widehat{\mathbf{S}}_{\gamma}(y), y \in \mathbb{R}$, in $L^{2}\left(\mathbb{S}^{2}\right)$, whose kernel depends on the scalar product $t=\langle\xi, \eta\rangle$ of the arguments $\xi, \eta \in \mathbb{S}^{2}$ and has the form

$$
\widehat{S}_{\gamma}(t ; y)=-(2 \pi)^{-1} u \frac{\sinh [y \arccos t]}{\sqrt{1-s^{2} t^{2}} \sinh (\pi y)} .
$$

For $v>0$, define

$$
\mathcal{U}(\nu ; \gamma)=(4 \pi)^{-1} \int_{-\infty}^{\infty} n\left(\nu, \widehat{\mathbf{S}}_{\gamma}(y)\right) d y, \quad v>0 .
$$

The function $\mathcal{U}(\nu ; \gamma)$ is very important for the proof of the existence of the Efimov effect. Denote $\mathscr{U}_{0}(\gamma)=\mathscr{U}(1 ; \gamma)$.

Similarly to $[8]$, we can derive that

$$
\begin{aligned}
\mathscr{U}_{0}(\gamma) & =\frac{1}{4 \pi} \int_{-\infty}^{\infty} n\left(1, \widehat{\mathbf{S}}_{\gamma}(y)\right) d y \\
& =\frac{1}{4 \pi} \sum_{l=0}^{\infty}(2 l+1) \int_{-\infty}^{\infty} n\left(1, \widehat{\mathbf{S}}_{\gamma}^{(l)}(y)\right) d y,
\end{aligned}
$$

where $\widehat{\mathbf{S}}_{\gamma}^{(l)}(y)$ is the multiplication operator by number

$$
\widehat{S}_{\gamma}^{(l)}(y)=2 \pi \int_{-1}^{1} P_{l}(t) \widehat{S}_{\gamma}(t ; y) d t
$$

in $\mathfrak{G}_{l}$ the subspace of the harmonics of degree $l$ and $P_{l}(t)$ are Legendre polynomials. It follows from (83) and Lemma 19 that

$$
\begin{aligned}
& \mathscr{U}_{0}(\gamma) \\
& \quad \geq \frac{1}{4 \pi} \int_{-\infty}^{+\infty} n\left(1, \widehat{\mathbf{S}}_{\gamma}^{(1)}(y)\right) d y=\frac{3}{4 \pi} \operatorname{mes}\left\{x: \widehat{S}_{\gamma}^{(1)}(x)>1\right\} .
\end{aligned}
$$

Lemma 19. The following assertions are true.

(a) $\widehat{S}_{\gamma}^{(0)}(y)<0$.

(b) $\sup _{y} \widehat{S}_{\gamma}^{(1)}(y)>1, \gamma>\gamma^{*}$, and $\sup _{y} \widehat{S}_{\gamma}^{(1)}(y)<1, \gamma<\gamma^{*}$.

(c) $\widehat{S}_{\gamma}^{(2)}(y)<0, \gamma>0$, and for any $\gamma, \gamma<\gamma^{*}$ there exists a positive number $\varepsilon=\varepsilon_{\gamma}$ such that $\sup _{y} \widehat{S}_{\gamma}^{(1)}(y)<1-\varepsilon_{\gamma}$, $l \geq 3$.

Proof. For the completeness of the proof of this lemma we reproduce in Appendix A.

The positivity of $\mathscr{U}_{0}(\gamma)$ follows from the fact that mes $\{x$ : $\left.\widehat{S}_{\gamma}^{(1)}(x)>1\right\}>0$, if $\gamma>\gamma^{*}$, which is proved in Lemma 19. and

We remark that for all $\gamma>\gamma_{0}$ the number $\mathscr{U}_{0}(\gamma)$ is finite

$$
\begin{aligned}
\mathscr{U}_{0}(\gamma) \leq & \frac{3}{\pi} \frac{\log (\pi((1+\gamma) / \sqrt{1+2 \gamma}))}{\pi-2 \arcsin (\gamma /(1+\gamma))} \\
& \times\left[\frac{\log \left(16(1+\gamma) / \pi^{1 / 2} 3^{3 / 4} \sqrt{1+2 \gamma}\right)}{\log ((1+\gamma) / \gamma+\sqrt{1+2 \gamma} / \gamma)}+1\right]^{2} .
\end{aligned}
$$

The difference of the operators $S_{\mathbf{r}}$ and $T_{1}(r)$ is compact (up to unitarily equivalence) and hence, taking into account that $\mathbf{r}=|1 / 2| \log \left|E_{\min }-z\right|$ and Lemma 17, we obtain the limit (80).

Proof of Part (ii) of Theorem 10. Theorem 18 together with Lemmas 13,16, and 17 complete the proof of Theorem 10.

\subsection{The Finiteness of the Discrete Spectrum of $H$.}

Lemma 20. Let $\gamma<\gamma^{*}$ and the hypothesis of part (i) of Theorem 10 be fulfilled. Then there exists a number $\epsilon=\epsilon_{\gamma}$ depending on $\gamma$, such that

$$
\sup \sigma_{\text {ess }}\left(T\left(E_{\min }\right)\right)<1-\epsilon_{\gamma}
$$

Proof. See Appendix B.

Remark 21. In the proof of Lemma 20, we can see that the main part of the operator $T\left(E_{\min }\right)$ is unitarily equivalent to the direct sum of multiplication operators in $L^{2}(\mathbb{R})$ by the functions $\widehat{S}_{\gamma}^{(0)}(\cdot), l=0,1, \ldots$, in Lemma 19 . So $T\left(E_{\min }\right)$ is not compact operator.

By virtue of Lemma 20 the operator $T\left(E_{\min }\right)$ cannot have many eigenvalues larger than 1 , and hence

$$
n\left(1, T\left(E_{\min }\right)\right)<\infty \text {. }
$$

This inequality and Lemma 15 complete the proof of part (i) of Theorem 10. 


\section{Appendices}

\section{A. Proof of Lemma 19}

Case $(l=0)$. By $(83)$ we first calculate $\widehat{S}_{\gamma}^{(0)}(y)$ as

$$
\widehat{S}_{\gamma}^{(0)}(y)=-\frac{u}{\sinh (\pi \gamma)} \int_{-1}^{1} \frac{\sinh [y(\arccos (s t))]}{\sqrt{1-s^{2} t^{2}}} d t .
$$

In $[8]$ it is proven that for any $y \in \mathbb{R}$,

$$
\begin{aligned}
& \frac{u \sinh [y(\arcsin s)]}{s y \cosh (\pi y)} \\
& \quad=\frac{u}{\sinh (\pi \gamma)} \int_{-1}^{1} \frac{\sinh [y(\arccos (s t))]}{\sqrt{1-s^{2} t^{2}}} d t .
\end{aligned}
$$

Since this number is positive by (A.1) we have $\widehat{S}_{\gamma}^{(0)}(y)<0$ for all $y \in \mathbb{R}$.

Case $(l=1)$. For any $y \in \mathbb{R}^{1}$, the $\widehat{S}_{\gamma}^{(1)}(y)$ can be written in the form

$$
\widehat{S}_{\gamma}^{(1)}(y)=\frac{u}{\sinh (\pi y / 2)} \int_{0}^{1} \frac{t \sinh [y(\arcsin (s t))]}{\sqrt{1-s^{2} t^{2}}} d t .
$$

Since the integrand in (A.3) is positive, we obtain that the function $\widehat{S}_{\gamma}^{(1)}(y)$ is also positive and even as $y \in \mathbb{R}^{1}$. The function $\sinh \beta y / \sinh \alpha y$ is strictly decreased on $[0, \infty)$ for $\alpha>\beta$ and hence the function $\widehat{S}_{\gamma}^{(1)}(y)$ strictly decreases on $[0, \infty)$.

Let $b_{1}(\gamma)=\sup _{y} \widehat{S}_{\gamma}^{(1)}(y)$. Then we have

$$
b_{1}(\gamma)=\frac{2(1+\gamma)^{2}}{\pi \gamma \sqrt{1+2 \gamma}}-\frac{2(1+\gamma)^{2}}{\pi \gamma^{2}} \arcsin \frac{\gamma}{1+\gamma} .
$$

Since $\widehat{S}_{\gamma}^{(1)}(y)>0$ for all $y \in \mathbb{R}^{1}$ and $\lim _{y \rightarrow \infty} \widehat{S}_{\gamma}^{(1)}(y)=0$, it follows from (A.3) and (A.4) that the range of the function $\widehat{S}_{\gamma}^{(1)}(y)$ coincides with the set $\left(0, b_{1}(\gamma)\right]$.

The function $b_{1}(\gamma)$ defined on $(0, \infty)$ is continuous and strictly increases.

Moreover,

$$
\lim _{\gamma \rightarrow 0} b_{1}(\gamma)=0, \quad \lim _{\gamma \rightarrow \infty} b_{1}(\gamma)=\infty .
$$

Thus the equation $b_{1}(\gamma)=1$ has a unique simple solution $\gamma_{0}>$ 0 and $b_{1}(\gamma)<1\left(b_{1}(\gamma)>1\right.$, resp.) for $\gamma<\gamma^{*}\left(\gamma>\gamma^{*}\right.$, resp.).

Case $(l=2)$. The function $\widehat{S}_{\gamma}^{(2)}(y)$ is calculated by

$$
\widehat{S}_{\gamma}^{(2)}(y)=-\frac{u}{2} \int_{0}^{1} \frac{3 t^{2}-1}{\sqrt{1-s^{2} t^{2}}} \frac{\cosh [y(\arcsin s t)]}{\cosh (\pi y / 2)} d t .
$$

It is easy to see that the inequalities

$$
\begin{aligned}
& \frac{\cosh [y(\arcsin s t)]}{\sqrt{1-s^{2} t^{2}}} \\
& <\frac{\cosh [y(\arcsin (1 / \sqrt{3}) s)]}{\sqrt{1-(1 / 3) s^{2}}}, \quad t \in\left[0, \frac{1}{\sqrt{3}}\right), \\
& \frac{\cosh [y(\arcsin s t)]}{\sqrt{1-s^{2} t^{2}}} \\
& >\frac{\cosh [y(\arcsin (1 / \sqrt{3}) s)]}{\sqrt{1-(1 / 3) s^{2}}}, \quad t \in\left(\frac{1}{\sqrt{3}}, 1\right],
\end{aligned}
$$

hold.

Then using the inequalities (A.7), we obtain the following inequality:

$$
\begin{aligned}
& \widehat{S}_{\gamma}^{(2)}(y) \\
& \quad<-\frac{u}{2} \frac{\cosh [y(\arcsin (1 / \sqrt{3}) s)]}{\sqrt{1-(1 / 3) s^{2}} \cosh (\pi y / 2)} \int_{0}^{1}\left(3 t^{2}-1\right) d t=0 .
\end{aligned}
$$

From here it follows that for all $\gamma>0$

$$
\sup \sigma\left(\widehat{S}_{\gamma}^{(2)}(y)\right) d y \leq 0 .
$$

Case ( $l \geq 3)$. By Lemma 3.2 of [8], we get that

$$
\begin{aligned}
\left|\widehat{S}_{\gamma}^{(l)}(y)\right| & <F(l ; \gamma) \\
& =\frac{16}{\pi^{1 / 2}[3(2 l+1)]^{3 / 4}} \frac{1+\gamma}{\sqrt{1+2 \gamma}}\left(\frac{\gamma}{1+\gamma+\sqrt{1+2 \gamma}}\right)^{l}
\end{aligned}
$$

for all $l \geq 0$ and $\gamma>0$.

Since the function $F(l ; \cdot)$ is increasing and the $F(\cdot ; \gamma)$ is decreasing, we have

$$
\sup _{y}\left|\hat{S}_{\gamma}^{(l)}(y)\right|<F\left(l ; \gamma^{*}\right)<F\left(3 ; \gamma^{*}\right)<F(3 ; 16)<1
$$

for any $\gamma \leq \gamma^{*}$ and $l \geq 3$.

Thus for any $\gamma \geq 0$ and $y \in \mathbb{R}^{1}$ we have $\widehat{S}_{\gamma}^{(l)}(y) \leq 0, l=0,2$ and for any $\gamma<\gamma^{*}$ (for any $\gamma \geq 0$ and $l \geq 3$, resp.) we have $\left|\widehat{S}_{\gamma}^{(1)}(y)\right|<1$ (resp. $\left.\left|\widehat{S}_{\gamma}^{(l)}(y)\right|<1\right)$.

These facts complete the proof of the lemma.

\section{B. Proof of Lemma 20}

Since the operator $T\left(E_{\min }\right)-T(\delta ; 0)$ is Hilbert-Schmidt (see Lemma 16), Weyl's Theorem implies that $\sigma_{\text {ess }}\left(T\left(E_{\min }\right)\right)=$ $\sigma_{\text {ess }}(T(\delta ; 0))$.

The space $L^{2}\left(\widehat{U}_{\delta}(0)\right)$ of all functions $w(p)$ having support in $\widehat{U}_{\delta}(0)=\left\{p \in \mathbb{T}^{3}:\left|W^{1 / 2} p\right|<\delta\right\}$ is an invariant subspace of the operator $T_{\gamma}(\delta ; 0)$. 
Let $\widehat{T}_{\gamma}(\delta ; 0)$ be the restriction of the operator $T_{\gamma}(\delta ; 0)$ to the invariant subspace $L^{2}\left(\widehat{U}_{\delta}(0)\right)$.

The operator $\widehat{T}_{\gamma}(\delta ; 0)$ is unitary equivalent to the integral operator $\mathbf{S}_{\gamma}$ acting in $L^{2}(0,+\infty) \otimes L^{2}\left(\mathbb{S}^{2}\right)$, with the kernel $S_{\gamma}\left(x-x^{\prime} ;<\xi, \eta>\right), \xi, \eta \in \mathbb{S}^{2}$, where

$$
\begin{gathered}
S_{\gamma}(x ; t)=-(2 \pi)^{-2} \frac{u}{\cosh x+s t}, \quad u=\frac{1+\gamma}{\sqrt{1+2 \gamma}}, \\
s=\frac{\gamma}{1+\gamma} .
\end{gathered}
$$

The equivalence is given by the unitary operator $M$ : $L^{2}\left(U_{\delta}(0)\right) \rightarrow L^{2}\left((0, \infty) \otimes L^{2}\left(\mathbb{S}^{2}\right)\right)$, where

$$
(M f)(x, w)=\delta^{3 / 2} e^{-3 x / 2} f\left(\delta e^{-x} W \frac{1}{2} w\right), \quad w \in \mathbb{S}^{2} .
$$

Therefore, the essential spectrum of $T_{\gamma}^{(0)}(\delta)$ and $\mathbf{S}_{\gamma}$ coincide.

Similarly to [8], we have

$$
\mathbf{S}_{\gamma}=\sum_{l=0}^{\infty} \oplus\left(\mathbf{S}_{\gamma}^{(l)} \otimes \mathscr{P}_{l}\right),
$$

where $\mathscr{P}_{l}: L^{2}\left((0, \infty) \otimes L^{2}\left(\mathbb{S}^{2}\right)\right) \rightarrow \mathscr{G}_{l}$ is the orthogonal projector onto $\mathfrak{G}_{l}$, the operator $\mathbf{S}_{\gamma}^{(l)}$ is operator in $L^{2}(0, \infty)$ with the kernel

$$
S_{\gamma}^{(l)}\left(x-x^{\prime}\right)=2 \pi \int_{-1}^{1} P_{l}(t) S_{\gamma}\left(x-x^{\prime} ; t\right) d t .
$$

Now, comparing the definition (81) and (B.1) and applying the equality

$$
\frac{\sinh (y \theta)}{\sin \theta \sinh (\pi y)}=\frac{1}{2 \pi} \int_{-\infty}^{\infty} e^{-i y x} \frac{d x}{\cosh x+\cos \theta}, \quad 0<\theta<\pi,
$$

we see that

$$
\widehat{S}_{\gamma}(t ; y)=\int_{-\infty}^{\infty} e^{-i y x} S_{\gamma}(x ; t) d x
$$

By (B.1) and (B.4), this yields

$$
\widehat{S}_{\gamma}^{(l)}(y)=\int_{-\infty}^{\infty} e^{-i y x} S_{\gamma}^{(l)}(x) d x .
$$

One can check that the functions in (84) and (B.7) coincide.

Since the operator $\mathbf{S}_{\gamma}^{(l)}$ is of convolution type, we conclude that it is unitarily equivalent to the operators $\widehat{\mathbf{S}}_{\gamma}^{(l)}$ of multiplication by functions $\widehat{S}_{\gamma}^{(l)}(y)$ in the space $L^{2}\left(\mathbb{R}^{1}\right)$. Therefore,

$$
\sigma\left(\widehat{\mathbf{S}}_{\gamma}^{(l)}\right)=\sigma\left(\widehat{\mathbf{S}}_{\gamma}^{(l)}\right)=\operatorname{Ran}\left(\widehat{S}_{\gamma}^{(l)}(y)\right)
$$

Recall that the number $\gamma^{*}$ is the unique solution of the equation $b_{1}(\gamma)=1$.
As it was mentioned in Lemma 19, for any $\gamma<\gamma^{*}$ there exists the number $\epsilon_{\gamma}>0$ such that for all $l \geq 0$

$$
\sup _{y}\left|\widehat{S}_{\gamma}^{(l)}(y)\right|<1-\epsilon_{\gamma}
$$

The equality (B.3), (B.8), and (B.9) proved the inequality in the lemma.

\section{Acknowledgments}

This work was partially supported by the University Putra Malaysia under research university grant scheme (RUGS), and project code is 9190883 . This work is also partially supported by the Fundamental Science Foundation of Uzbekistan. The authors are indebted to the anonymous referees for a number of constructive comments.

\section{References}

[1] V. Efimov, "Energy levels of three resonantly interacting particles," Nuclear Physics A, vol. 210, pp. 157-158, 1973.

[2] S. Albeverio, R. Høegh-Krohn, and T. T. Wu, "A class of exactly solvable three-body quantum mechanical problems and the universal low energy behavior ", Physics Letters A, vol. 83, no. 3, pp. 105-109, 1981.

[3] S. Albeverio, S. N. Lakaev, and K. A. Makarov, "The Efimov effect and an extended Szegö-Kac limit theorem," Letters in Mathematical Physics, vol. 43, no. 1, pp. 73-85, 1998.

[4] R. D. Amado and J. V. Noble, “On efimov's effect: a new pathology of three-particle systems," Physics Letters B, vol. 35, no. 1, pp. 25-27, 1971.

[5] G. F. Dell'Antonio, R. Figari, and A. Teta, "Hamiltonians for systems of $N$ particles interacting through point interactions," Annales de l'Institut Henri Poincaré, vol. 60, no. 3, pp. 253-290, 1994.

[6] L. D. Faddeev and S. P. Merkuriev, Quantum Scattering Theory for Several Particle Systems, Kluwer Academic, New York, NY, USA, 1993.

[7] Yu. N. Ovchinnikov and I. M. Sigal, "Number of bound states of three-body systems and Efimov's effect," Annals of Physics, vol. 123, no. 2, pp. 274-295, 1979.

[8] A. V. Sobolev, "The Efimov effect. Discrete spectrum asymptotics," Communications in Mathematical Physics, vol. 156, no. 1, pp. 101-126, 1993.

[9] H. Tamura, "The Efimov effect of three-body Schrödinger operators," Journal of Functional Analysis, vol. 95, no. 2, pp. 433$459,1991$.

[10] H. Tamura, "Asymptotics for the number of negative eigenvalues of three-body Schrödinger operators with Efimov effect," in Spectral and Scattering Theory and Applications, vol. 23 of Advanced Studies in Pure Mathematics, pp. 311-322, Mathematical Society of Japan, Tokyo, Japan, 1994.

[11] D. R. Jafaev, "On the theory of the discrete spectrum of the three-particle schridinger operator," Mathematics of the USSRSbornik, vol. 23, no. 4, pp. 535-559, 1974.

[12] X. P. Wang, "On the existence of the N-body Efimov effect," Journal of Functional Analysis, vol. 209, no. 1, pp. 137-161, 2004.

[13] R. A. Minlos and H. Spohn, "The three-body problem in radioactive decay: the case of one atom and at most two 
photons," in Topics in Statistical and Theoretical Physics, vol. 177 of Transactions of the American Mathematical Society Series 2, pp. 159-193, American Mathematical Society, Providence, RI, USA, 1996.

[14] V. A. Malyshev and R. A. Minlos, Linear Infinite-Particle Operators (Translations of Mathematical Monographs), vol. 143, American Mathematical Society, Providence, RI, USA, 1995.

[15] D. C. Mattis, “The few-body problem on a lattice," Reviews of Modern Physics, vol. 58, no. 2, pp. 361-379, 1986.

[16] A. I. Mogilner, "Hamiltonians in solid-state physics as multiparticle discrete Schrödinger operators: problems and results," in Many-particle Hamiltonians: Spectra and Scattering, vol. 5 of Advances in Soviet Mathematics, pp. 139-194, American Mathematical Society, Providence, RI, USA, 1991.

[17] C. Buhler, S. Yunoki, and A. Moreo, "Magnetic domains and stripes in a spin-fermion model for cuprates," Physical Review Letters, vol. 84, no. 12, pp. 2690-2693, 2000.

[18] K. O. Friedrichs, Perturbation of Spectra in Hilbert Space, American Mathematical Society, Providence, RI, USA, 1965.

[19] A. M. Mel'nikov, "On the lower branches of the spectrum of two fermions interacting with a boson gas (exiton)," Russian Mathematical Surveys, vol. 50, no. 4, pp. 824-825, 1995.

[20] I. M. Sigal, A. Soffer, and L. Zielinski, "On the spectral properties of Hamiltonians without conservation of the particle number," Journal of Mathematical Physics, vol. 43, no. 4, pp. 1844-1855, 2002.

[21] Yu. V. Zhukov and R. A. Minlos, "The spectrum and scattering in the "spin-boson" model with at most three photons," Theoretical and Mathematical Physics, vol. 103, no. 1, pp. 398-411, 1995.

[22] S. Albeverio, S. N. Lakaev, and T. H. Rasulov, "On the spectrum of an hamiltonian in fock space. Discrete spectrum asymptotics," Journal of Statistical Physics, vol. 127, no. 2, pp. 191-220, 2007.

[23] S. Albeverio, S. N. Lakaev, and T. H. Rasulov, “The Efimov effect for a model operator associated with the Hamiltonian of a non conserved number of particles," Methods of Functional Analysis and Topology, vol. 13, no. 1, pp. 1-16, 2007.

[24] S. Albeverio, S. N. Lakaev, and Z. I. Muminov, "Schrödinger operators on lattices. The Efimov effect and discrete spectrum asymptotics," Annales Henri Poincaré, vol. 5, no. 4, pp. 743-772, 2004.

[25] S. N. Lakaev, "The efimov's effect of a system of three identical quantum iattice particles," Funktsional'nyi Analiz i Ego Prilozheniya, vol. 27, no. 3, pp. 15-28, 1993, translated in Functional Analysis and Its Applications.

[26] G. F. Dell'Antonio, Z. I. Muminov, and Y. M. Shermatova, "On the number of eigenvalues of a model operator related to a system of three particles on lattices," Journal of Physics A, vol. 44, no. 31, Article ID 315302, 2011.

[27] D. S. Petrov, "Three-body problem in Fermi gases with shortrange interparticle interaction," Physical Review A, vol. 67, no. 1, Article ID 010703, 2003. 


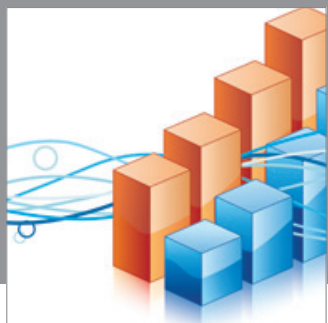

Advances in

Operations Research

mansans

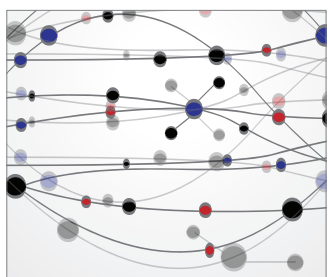

The Scientific World Journal
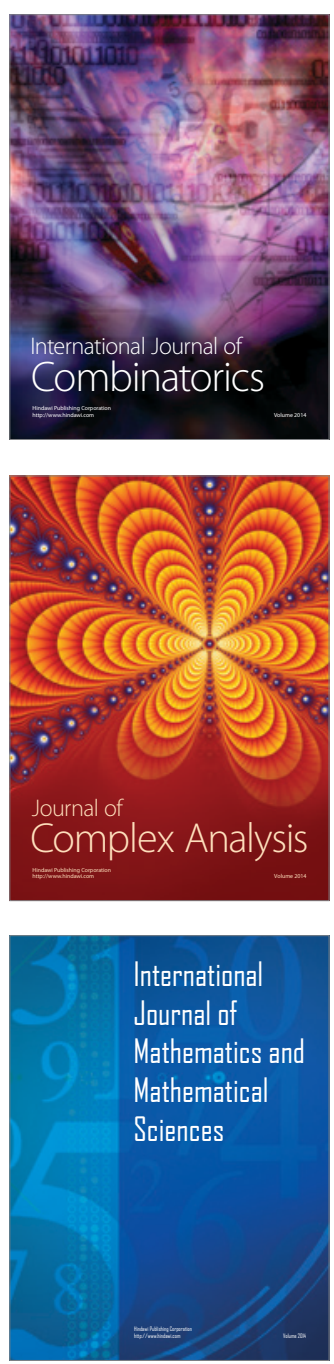
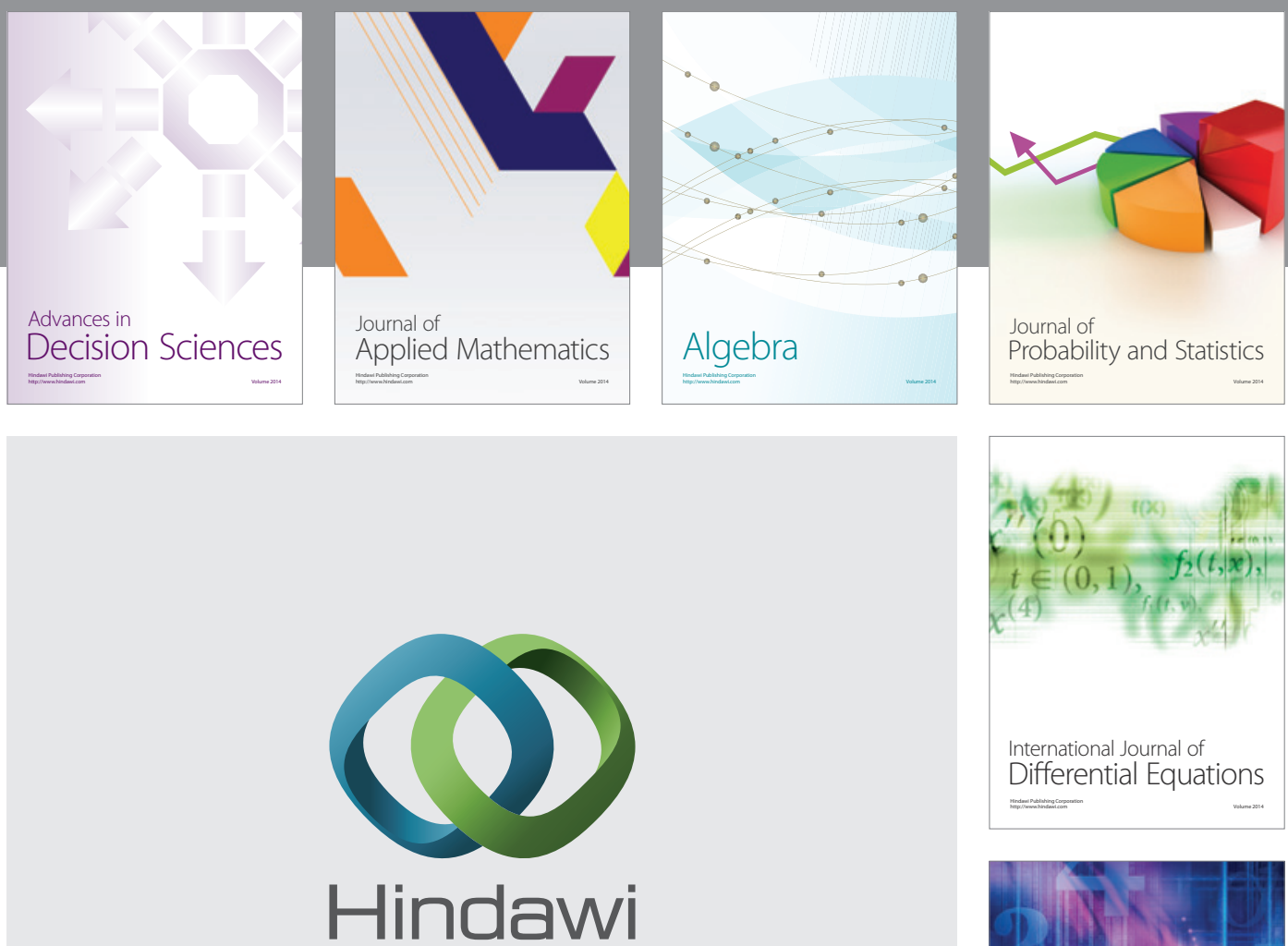

Submit your manuscripts at http://www.hindawi.com
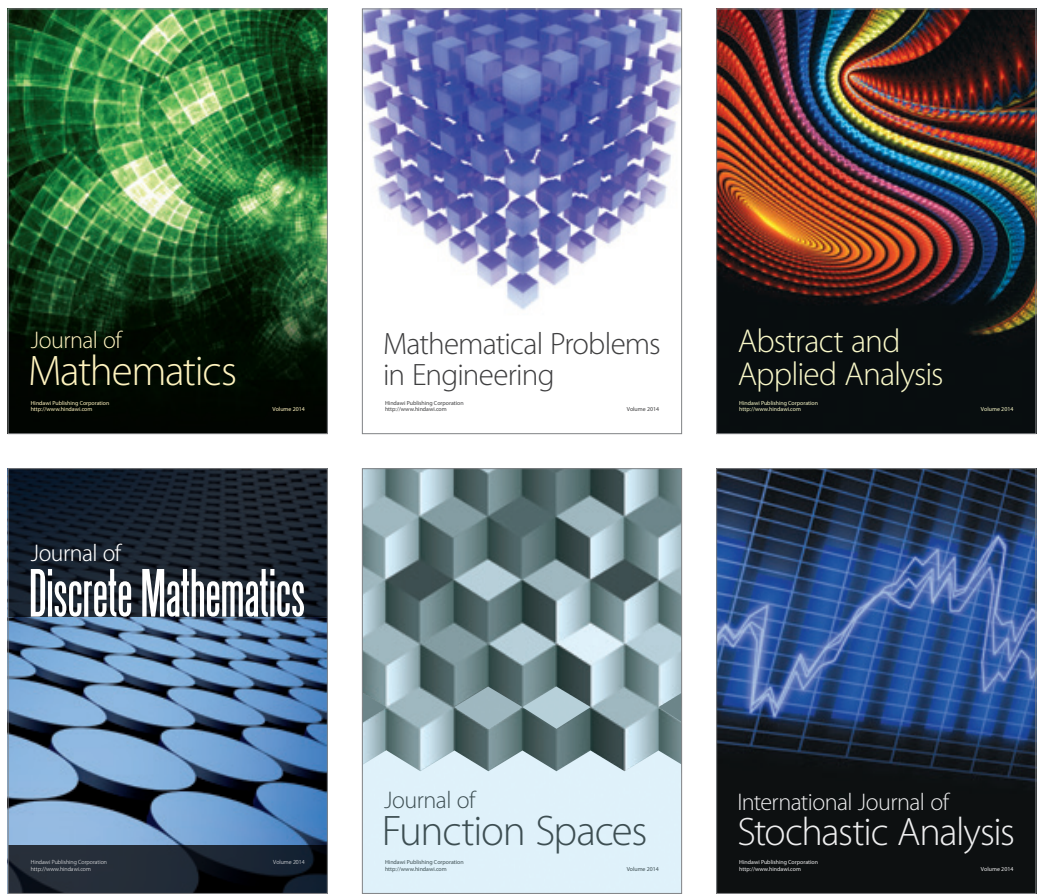

Journal of

Function Spaces

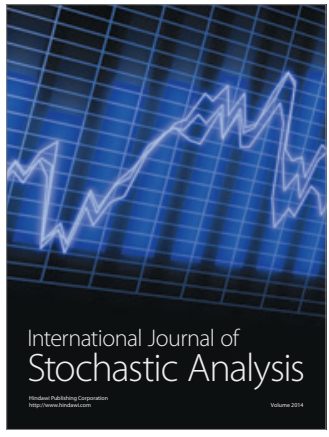

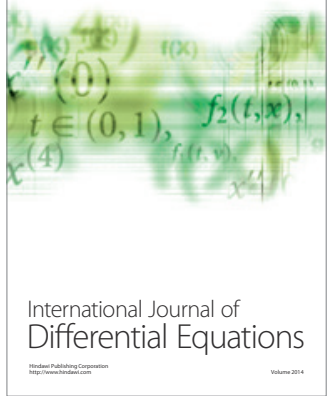
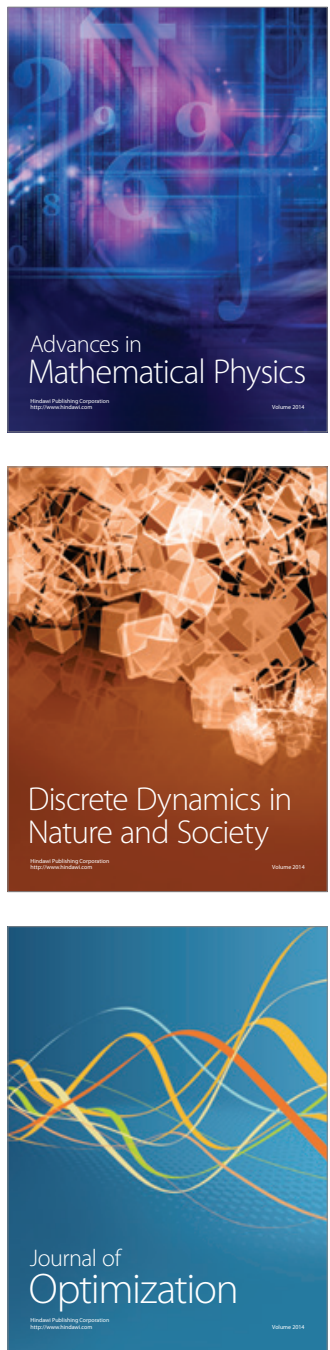